\title{
Counseling about gestational weight gain and healthy lifestyle during pregnancy: Canadian maternity care providers' self-evaluation
}

This article was published in the following Dove Press journal:

International Journal of Women's Health

27 September 2013

Number of times this article has been viewed

\section{Zachary M Ferraro' \\ Kaitlin S Boehm' \\ Laura M Gaudet ${ }^{2,3}$ \\ Kristi B Adamo ${ }^{1,4,5}$}

'Healthy Active Living and Obesity Research Group, Children's Hospital of Eastern Ontario Research Institute, Ottawa, Ontario, Canada; ${ }^{2}$ Horizon Health Network, Saint John, New Brunswick, Canada; ${ }^{3}$ Department of Obstetrics and Gynaecology, Faculty of Medicine, Dalhousie University, Halifax, Nova Scotia, Canada; ${ }^{4}$ School of Human Kinetics, Faculty of Health Sciences, ${ }^{5}$ Department of Pediatrics, Faculty of Medicine, University of Ottawa, Ottawa, Ontario, Canada
Correspondence: Kristi B Adamo Healthy Active Living and Obesity (HALO) Research Group, Children's Hospital of Eastern Ontario Research Institute, 40I Smyth Road, Ottawa, Ontario, Canada KIH8LI

Tel +l 6137377600 ext 4190

Fax +I 6137384800

Email kadamo@cheo.on.ca
Introduction: There is discord between the recall of maternity care providers and patients when it comes to discussion of gestational weight gain (GWG) and obesity management. Few women report being advised on GWG, physical activity (PA), and nutrition, yet the majority of health care providers report discussing these topics with patients. We evaluated whether various Canadian maternal health care providers can identify appropriate GWG targets for patients with obesity and determine if providers report counseling on GWG, physical activity, and nutrition.

Methods: A valid and reliable e-survey was created using SurveyMonkey software and distributed by the Society of Obstetricians and Gynaecologists of Canada listserve. A total of 174 health care providers finished the survey. Respondents self-identified as general practitioners, obstetricians, maternal-fetal medicine specialists, midwives, or registered nurses.

Results: GWG recommendations between disciplines for all body mass index categories were similar and fell within Health Canada/Institute of Medicine (IOM) guidelines. Of those who answered this question, 110/160 (68.8\%) were able to correctly identify the maximum IOM GWG recommended for patients with obesity, yet midwives tended to recommend $0.5-1 \mathrm{~kg}$ more GWG $(P=0.05)$. PA counseling during pregnancy differed between disciplines $(P<0.01)$, as did nutrition counseling during pregnancy $(P<0.05)$.

Conclusion: In contrast to patient reports, the majority of health care providers document counseling on GWG, PA, and nutrition and appropriately identify GWG limits for obese patients. However, the content and quality of the discourse between patient and provider warrants further investigation.

Keywords: gestational weight gain, nutrition, obesity, physical activity, pregnancy

\section{Introduction}

The adverse effects of maternal pregravid obesity and excessive gestational weight gain (GWG) for short- and long-term health of both mother and offspring are well documented (as reviewed by Adamo et al). ${ }^{1}$ Although negative associations are clearly known, the path of knowledge translation from research literature to health care provider to patient has not been studied extensively. What is certain is that a significant number of at-risk patients are not able to correctly identify their target GWG - nearly $45 \%$ of newly pregnant overweight or obese women identify a GWG that exceeds IOM recommendations, which are based on pregravid BMI. ${ }^{2}$ The reasons behind this are complex, but the involvement of maternity care providers in knowledge provision is believed to be essential.

In a survey of pregnant women and maternity care providers from Ontario, Canada, it was concluded that there was a significant difference between patient- and 
physician-reported discussion. ${ }^{3}$ In their cross-sectional study of 42 obstetricians, midwives, and family medicine care providers, $95 \%$ reported counseling women to gain a specific amount of weight while $81 \%$ reported that they recommend values within the $2009 \mathrm{IOM} /$ Health Canada guidelines. ${ }^{3}$ Overall, these findings were incongruent with previously published information ${ }^{5}$ on patients' reports of counseling that suggested that a minority of patients $(<12 \%)$ report being counseled on appropriate GWG during pregnancy. It is possible that disrupted, insufficient, or inaccurate knowledge transfer may be related to a patient's inability to meet GWG recommendations during pregnancy. ${ }^{4,5}$ Furthermore, research has shown that the majority of health care providers report counseling their pregnant patients on appropriate GWG and physical activity recommendations, ${ }^{3,6}$ but that only two-thirds of all pregnant women report receiving information about physical activity or nutrition. ${ }^{7}$ However, less than half of all women, including those who are overweight or obese, report being accurately counseled on these topics. ${ }^{2,5}$ Given the double burden of pregravid obesity and potential adverse effects of excessive GWG, appropriate knowledge exchange concerning a modifiable risk factor (ie, GWG) to patients with obesity is of utmost importance.

There are several likely explanations for the discrepancy between patient- and provider-reported counseling. Interestingly, one study suggested that $40 \%-80 \%$ of information exchanged by health care providers is immediately forgotten by patients and up to $50 \%$ is remembered incorrectly. ${ }^{8}$ Further, although $80 \%$ of health care professionals' recommendations are within IOM guidelines, ${ }^{3}$ only a small minority of patients report that their care provider discussed the risks of excessive weight gain. ${ }^{5}$ Phelan et $\mathrm{al}^{2}$ showed that overweight and obese women have an 18-fold increase in the odds of being advised to over-gain - a clinically relevant problem given the additive risk of pregravid obesity and excess GWG. In addition, almost $70 \%$ of women use information sources other than their health care provider for advice on weight gain and nutrition in pregnancy. ${ }^{5}$

Systematic reviews support the clinical efficacy of nutrition and/or physical activity counseling during pregnancy on GWG management and maternal-fetal outcomes. ${ }^{910}$ For information transfer regarding GWG and obesity management to be successful, the care provider must supply correct information in an individualized manner and the patient must retain that information. Since, ideally, maternity care providers must correctly identify GWG targets and impart knowledge about healthy lifestyle during pregnancy, we elected to ask care providers directly about their information transfer. The main purpose of this study was to nationally evaluate whether maternal health care providers are able to correctly identify appropriate GWG for patients with obesity. We hypothesized that maternal health care providers would overreport recommended GWG limits for patients with obesity. Further, this study sought to determine providers' self-reported level of counseling regarding GWG, physical activity, and nutrition during pregnancy. We also aimed to determine whether there was a difference in self-reported levels of knowledge between disciplines of maternity care providers.

\section{Methods}

A novel survey was created using standard survey methodology, including design and review by content experts. It was then tested for validity and reliability and revised appropriately. To assess validity, the questionnaire for this study was designed by a team of researchers, including three content experts and one survey methodologist. Once consensus was reached among these individuals, the instrument was provided to three additional content experts to ensure the questionnaire addressed the questions of interest. All three experts provided suggestions for modification based on their assessment of content and construct validity. Suggestions were incorporated where acceptable, with disagreements discussed until consensus was reached between the lead author and the expert in question. The final questionnaire was then reviewed by all original team members and the three experts with no further revisions suggested. In order to assess reliability and reproducibility of the construct, ten individuals were asked to complete the questionnaire on two occasions, 4 weeks apart. Seven of the ten individuals returned two completed questionnaires, thus allowing inclusion in the analysis. The intra-observer variability for the majority of questions was within acceptable limits (proportion with agreement $>0.75$ ). Less than optimal variability was identified in several domains that dealt with practice patterns. In these domains, there was a general trend towards giving more "correct" answers. We believe that this indicates that study participants either achieved a greater level of knowledge or a greater level of awareness of their attitudes and practice patterns prior to completing the questionnaire a second time. While this renders our reproducibility less than ideal, it is, nonetheless, encouraging from a care-provision standpoint. As this is a cross-sectional survey, we are interested in the answers from the first questionnaire, which reflect attitudes and practice patterns currently in existence. The differences in the answers on the second questionnaire are, therefore, not concerning. 
SurveyMonkey ${ }^{\circledR}$ software (SurveyMonkey ${ }^{\circledR}$, Portland, OR, USA) was used to develop an electronic version of the survey that was distributed by the Society of Obstetricians and Gynaecologists of Canada (SOGC) to its member mailing list in May 2012 with follow-up occurring until study completion in August 2012. All members of the SOGC who agreed to accept surveys and could read English were eligible to participate. Responses were automatically collected and stored in SurveyMonkey and a reminder email was sent 2 weeks after the initial distribution. The survey remained open and active for 1 month. Respondents were asked to self-identify their role in provision of maternity care as general practitioners (GPs), obstetricians (OBs), maternal-fetal medicine specialists (MFMs [eg, perinatologists]), midwives (MWs), registered nurses (RNs), or "other" (which none selected, so this category is not present in the tables). No identifying information was collected and consent was implied by survey completion. The raw data were entered into and analyzed in SPSS (v 20; IBM Corporation, Armonk, NY, USA). Differences in response by provider role were compared using $t$-tests, analysis of variance (ANOVA), or chi-squared for homogeneity of proportions where appropriate. The survey was distributed to the SOGC emailing list, which included a total of 1,200 members who agreed to accept survey invitations. A sample size of 150 was required to determine the proportion of care providers who could correctly identify the upper limit of recommended GWG for obesity. This sample size was selected to estimate the proportion of care providers who could correctly identify the upper limit of GWG for women with obesity to within $\pm 7.5 \%$. Given that precision is worst (ie, widest $95 \%$ confidence intervals) at a probability value of 0.5 , and assuming a $20 \%$ response rate, 150 respondents were required to achieve the desired precision of $\pm 7.5 \%$. This provides a conservative estimate of precision when analyzing provider responses $(P \leq 0.05)$. The survey was approved by the Ottawa Hospital Research Ethics Board (OHREB Protocol \#20009937-01H).

\section{Results}

A total of 174 maternity care providers finished the survey. Table 1 shows the distribution of respondents by self-reported discipline. Table 2 reviews the IOM and SOGC guidelines for GWG and identifies those who recommend at, below, or above the accepted value of gain for pregnancy complicated by obesity. Of respondents who answered this question, $110 / 160(69 \%)$ indicated that they recommended at or below the maximum IOM GWG for patients with obesity. When disciplines were compared, there were some notable differences in GWG recommendations. Significant discrepancies
Table I Distribution of respondents by self-reported discipline

\begin{tabular}{lll}
\hline Discipline & $\begin{array}{l}\text { Number of } \\
\text { respondents }\end{array}$ & $\begin{array}{l}\text { \% of total } \\
\text { respondents }\end{array}$ \\
\hline GP & 29 & 16.7 \\
OB & 86 & 49.4 \\
MFM & 22 & 12.6 \\
MW & 32 & 18.4 \\
RN & 5 & 2.9 \\
Total & 174 & 100 \\
\hline
\end{tabular}

Abbreviations: GP, general practitioner; MFM, maternal-fetal medicine specialist; MW, midwife; OB, obstetrician; RN, registered nurse.

between MWs and MFMs were evident for counseling of normal weight (NW) patients, with MWs recommending $1.46 \mathrm{~kg}$ more than MFMs $(P=0.028)$. When responses specific to obese patients were examined independently, on average, OBs tended to recommend lower GWG $(6.63 \mathrm{~kg})$ than MFMs $(8.05 \mathrm{~kg})(P=0.07)$. In general, however, the recommendations for the four BMI categories were fairly consistent across the disciplines. Average health care provider-recommended GWG for obese patients fell below the IOM guideline upper limit of $9 \mathrm{~kg}$, but remained within the range (5-9 kg) for all disciplines. In keeping with SOGC guidelines ${ }^{19}$ (ie, $7 \mathrm{~kg}$ ), which are a consensus statement from Canada's obstetrical governing body, OBs recommend the least amount of GWG for obese mothers, at $6.63 \mathrm{~kg}$, while MWs recommend the highest gain of $8.48 \mathrm{~kg}$. Importantly, GWG recommendations for obesity ranged from $0.45-16.34 \mathrm{~kg}$.

Differences between disciplines occur with regard to counseling on GWG, physical activity, and nutrition. GPs and MWs reported counseling the most often, with $93.1 \%$ and $93.5 \%$, respectively, while only $73 \%$ of MFMs reported doing so $(P=0.07)$. Reported levels of physical activity counseling during pregnancy vary significantly between disciplines $(P=0.006)$. MWs reported the highest level of counseling, with $96.9 \%$ suggesting that they discuss physical activity to some extent, in contrast to $79.1 \%$ and $63.6 \%$ of OBs and MFMs, respectively. Similarly, differences exist with regards to nutrition counseling between disciplines $(P=0.03)$. GPs and MWs reported counseling more often, with $96.9 \%$ indicating that they do so, while $72.7 \%$ of MFMs reported making nutrition recommendations (Table 3).

Health care practitioners were then asked to answer a question regarding whether they feel they have adequate knowledge to counsel patients with respect to GWG, PA, and nutrition in pregnancy. A high percentage of all disciplines felt that they had adequate self-perceived knowledge pertaining to GWG. All disciplines reported similar confidence in their self-perceived knowledge to counsel on PA, 
Table 2 A comparison of IOM and SOGC gestational weight gain recommendations for pregravid obesity

\begin{tabular}{|c|c|c|c|c|c|c|c|}
\hline Discipline & $\begin{array}{l}\text { IOM max } \\
\text { recommendation } \\
(\mathrm{kg})\end{array}$ & $\begin{array}{l}\text { Range } \\
\text { (min-max) } \\
(\mathrm{kg})\end{array}$ & $\begin{array}{l}\text { Mean } \\
(\mathrm{kg})\end{array}$ & $\begin{array}{l}\text { \% recommended } \\
\text { (x/IOM upper } \\
\text { limit) }\end{array}$ & $\begin{array}{l}\text { Below upper } \\
\text { recommendation } \\
\mathbf{n}(\%)\end{array}$ & $\begin{array}{l}\text { Precise upper } \\
\text { recommendation } \\
\mathbf{n}(\%)\end{array}$ & $\begin{array}{l}\text { Exceeds upper } \\
\text { recommendation } \\
\mathrm{n}(\%)\end{array}$ \\
\hline GP & 9 & $0.45-11.0$ & $7.4 I$ & 82.4 & $16(59.2)$ & $2(7.4)$ & $9(33.3)$ \\
\hline $\mathrm{OB}$ & 9 & $0.45-16.34$ & 6.63 & 73.7 & $62(77.5)$ & $0(0)$ & I8 (22.5) \\
\hline MFM & 9 & $2.72-11.80$ & 8.05 & 89.5 & $13(68.4)$ & $0(0)$ & $6(31.6)$ \\
\hline MW & 9 & $0.45-14.07$ & 8.48 & 94.2 & $10(40.0)$ & $0(0)$ & $15(60.0)$ \\
\hline \multirow[t]{2}{*}{$\mathrm{RN}$} & 9 & $7.26-9.53$ & 8.40 & 93.3 & I $(50.0)$ & $0(0)$ & I (50.0) \\
\hline & $\begin{array}{l}\text { SOGC max } \\
\text { recommendation } \\
(\mathrm{kg})\end{array}$ & $\begin{array}{l}\text { Range } \\
\text { (min-max) } \\
(\mathrm{kg})\end{array}$ & $\begin{array}{l}\text { Mean } \\
(\mathrm{kg})\end{array}$ & $\begin{array}{l}\text { \% recommended } \\
\text { (x/SOGC upper } \\
\text { limit) }\end{array}$ & $\begin{array}{l}\text { Below upper } \\
\text { recommendation } \\
\mathbf{n}(\%)\end{array}$ & $\begin{array}{l}\text { Precise upper } \\
\text { recommendation } \\
\mathbf{n}(\%)\end{array}$ & $\begin{array}{l}\text { Exceeds upper } \\
\text { recommendation } \\
\text { n (\%) }\end{array}$ \\
\hline$\overline{\mathrm{GP}}$ & 7 & $0.45-11.0$ & 7.41 & 106.0 & $9(33.3)$ & I (3.7) & $17(63.0)$ \\
\hline OB & 7 & $0.45-16.34$ & 6.63 & 94.7 & $30(37.5)$ & I (I.2) & $49(61.3)$ \\
\hline MFM & 7 & $2.72-11.80$ & 8.05 & 115.0 & $2(10.5)$ & $2(10.5)$ & I5 (79.0) \\
\hline MW & 7 & $0.45-14.07$ & 8.48 & 121.1 & $6(24.0)$ & $0(0)$ & $19(76.0)$ \\
\hline $\mathrm{RN}$ & 7 & $7.26-9.53$ & 8.40 & 120.0 & $0(0)$ & $0(0)$ & $2(100.0)$ \\
\hline
\end{tabular}

Abbreviations: GP, general practitioner; IOM, Institute of Medicine; MFM, maternal-fetal medicine specialist; MW, midwife; OB, obstetrician; RN, registered nurse; SOGC, Society of Obstetricians and Gynaecologists of Canada.

though few reported having specific training in this area (Table 4). Significant differences in self-perceived knowledge to counsel on nutrition existed between the disciplines $(P=0.03)$. GPs felt the most knowledgeable, with $93.1 \%$ reporting that they feel they have adequate self-perceived knowledge, whereas OB, MFM, and MW values were much lower $(69.8 \%, 54.5 \%$, and $71.9 \%$, respectively).

When asked to identify who should be providing information on GWG to patients, a test for homogeneity of proportions between disciplines found no differences between the health care provider groups $(P \leq 0.05)$. The majority of respondents $(98.62 \%)$ from all disciplines felt that maternity care providers (GPs, OBs, MWs, etc) should be providing this information, as opposed to public health messaging and allied care providers (Table 5); this is important since GPs are the point of first contact in clinical care when a women is planning to become or is pregnant. However, fewer than $80 \%$ of all maternity health care providers feel that GPs should be solely responsible for exchanging GWG recommendations.

Table 3 Health care provider self-reported counseling of healthy lifestyle factors during prenatal visits

\begin{tabular}{lllllll}
\hline & GP & OB & MFM & MW & RN & $P$ \\
\hline GWG & 93.1 & 83.7 & 72.7 & 93.5 & 60.0 & 0.07 \\
& $(27 / 29)$ & $(72 / 86)$ & $(16 / 22)$ & $(29 / 31)$ & $(3 / 5)$ & \\
PA & 93.1 & 79.1 & 63.6 & 96.9 & 60.0 & 0.006 \\
& $(27 / 29)$ & $(68 / 86)$ & $(14 / 22)$ & $(31 / 32)$ & $(3 / 5)$ & \\
\multirow{4}{*}{ Nutrition } & 96.6 & 81.4 & 72.7 & 96.9 & 80.0 & 0.03 \\
& $(28 / 29)$ & $(70 / 86)$ & $(16 / 22)$ & $(31 / 32)$ & $(4 / 5)$ & \\
\hline
\end{tabular}

Note: Values provided as percent, with absolute number of responses in parentheses. Abbreviations: GP, general practitioner; GWG, gestational weight gain; MFM, maternal-fetal medicine specialist; MW, midwife; OB, obstetrician; PA, physical activity; RN, registered nurse.
Public health was the least selected option, with only $63.1 \%$ of all care providers identifying this domain as responsible for providing GWG information. In general, MFMs reported that all health professionals should be counseling on GWG.

\section{Discussion}

The primary aim of this study was to assess whether maternity care providers correctly identify the upper limit of acceptable gain in pregnancy complicated by obesity and to assess variation between different health care providers. To our knowledge, this is the first study to address this question by surveying maternal health care providers across Canada with respect to self-perceived knowledge of GWG, physical activity, and nutrition.

\section{Provider ability to correctly identify GWG recommendations}

Encouragingly, we did not identify any significant differences in maximum GWG recommendations between maternity care

Table 4 Providers' perceptions of having adequate self-perceived knowledge about GWG, PA, and nutrition

\begin{tabular}{llllll}
\hline & GP & OB & MFM & MW & RN \\
\hline GWG & 86.2 & 90.7 & 100.0 & 93.8 & 80.0 \\
& $(25 / 29)$ & $(78 / 86)$ & $(22 / 22)$ & $(30 / 32)$ & $(4 / 5)$ \\
PA & 89.3 & 89.4 & 95.5 & 84.4 & 100.0 \\
\multirow{4}{*}{ Nutrition } & $(25 / 28)$ & $(76 / 85)$ & $(21 / 22)$ & $(27 / 32)$ & $(5 / 5)$ \\
& 93.1 & 69.8 & 54.5 & 71.9 & 80.0 \\
& $(27 / 29)$ & $(60 / 86)$ & $(12 / 22)$ & $(23 / 32)$ & $(4 / 5)$ \\
\hline
\end{tabular}

Note: Values provided as percent, absolute number of responses in parentheses. Abbreviations: GP, general practitioner; GWG, gestational weight gain; MFM, maternal-fetal medicine specialist; MW, midwife; OB, obstetrician; PA, physical activity; RN, registered nurse. 
Table 5 Who do respondents believe is responsible for providing GWG recommendations?

\begin{tabular}{|c|c|c|c|c|c|}
\hline \multirow[t]{2}{*}{ Discipline } & \multirow{2}{*}{$\begin{array}{l}\text { Number of } \\
\text { respondents }\end{array}$} & \multicolumn{4}{|c|}{ Discipline(s) providers feel are responsible for giving GWG advice } \\
\hline & & $\begin{array}{l}\text { All maternity } \\
\text { care providers }\end{array}$ & GP & $\begin{array}{l}\text { Allied health } \\
\text { care professionals }\end{array}$ & $\begin{array}{l}\text { Public } \\
\text { health }\end{array}$ \\
\hline GP & 29 & $100(29 / 29)$ & $82.8(24 / 29)$ & $72.4(21 / 29)$ & $75.9(22 / 29)$ \\
\hline OB & 84 & $97.6(82 / 84)$ & $66.7(56 / 84)$ & $83.3(70 / 84)$ & $57.1(48 / 84)$ \\
\hline MFM & 22 & $95.5(21 / 22)$ & $95.5(21 / 22)$ & $100(22 / 22)$ & $72.7(16 / 22)$ \\
\hline MW & 32 & $100(32 / 32)$ & $75(24 / 32)$ & $78.1(25 / 32)$ & $50(16 / 32)$ \\
\hline RN & 5 & $100(5 / 5)$ & $80(4 / 5)$ & $80(4 / 5)$ & $60(3 / 5)$ \\
\hline Mean \% & & 98.62 & 80 & 82.76 & 63.14 \\
\hline
\end{tabular}

Notes: Results displayed as percentage of each discipline, with absolute number in parentheses. For example, row one shows that I00\% of GPs feel that all maternity care providers should make GWG recommendations to their pregnant patients, while $83 \%$ of GPs feel fellow GPs should provide this information and $72 \%$ of GPs believe that allied care professionals should disseminate this information.

Abbreviations: GP, general practitioner; GWG, gestational weight gain; MFM, maternal-fetal medicine specialist; MW, midwife; OB, obstetrician; RN, registered nurse.

providers, with all disciplines accurately reporting GWG within recommended thresholds for patients with obesity. This trend was observed when using both the IOM and SOGC recommendations, although using a lower GWG threshold (ie, $7 \mathrm{~kg}$ from SOGC) led to a greater number of providers recommending above current guidelines. This suggests that self-selected volunteers who completed the survey are well informed of the upper limits of acceptable GWG based on the IOM/Health Canada recommendations. A shortcoming of these recommendations is the inability to offer specific guidelines for higher BMIs within the obese category (ie, classes I, II, and III). For instance, it has been proposed that, in cases of super obesity (ie, BMI $>40$ ), virtually no gain may serve to limit adverse outcomes. ${ }^{11}$ Thus, having a single recommendation for the heterogeneous obese population may have led different providers to make recommendations based on the patient population most often presenting for care in their practice and not based on the individual patient risk of excessive gain. Recently, the Edmonton Obesity Staging System (EOSS) has been proposed as a novel way to stratify weight-related risk for patients with obesity presenting for clinical weight management based on the presence or absence of clinical risk factors as opposed to BMI alone, ${ }^{12}$ as BMI has been shown to be a poor indicator of health and mortality. ${ }^{13}$ Developing a tool of this nature or evaluating the EOSS in pregnancy complicated by obesity may aid in greater evidence-based GWG recommendations in such a heterogeneous population. Although not addressed in our study, the efficacy of quality GWG information transfer should be questioned, as a minority of patients report being appropriately counseled to gain within these recommendations ${ }^{4}$ and few patients report being given GWG-specific advice at all. ${ }^{7}$ Lastly, the providers in this sample globally recommended a value of GWG lower than the maximum, which may provide additional support for more conservative
GWG guidelines for the obese category and/or for basing GWG recommendations on factors other than BMI alone (ie, EOSS for pregnancy). ${ }^{11,14}$

\section{GWG, physical activity, and nutrition counseling}

In contrast to previous studies, ${ }^{5,7}$ the majority of respondents to our survey reported that they do counsel on GWG, physical activity, and nutrition to their pregnant patients. MWs and GPs appear to be the most active in this area. Despite the relatively high levels of self-reported counseling, the remaining portion of providers who do not counsel on GWG represent a large number of health care providers nationwide. Thus, a significant number of patients do not receive education about the negative health consequences of an inappropriate GWG or about the healthy behaviors that can help with weight management during pregnancy. ${ }^{2,715-17}$ This lack of knowledge is forcing many women to use resources other than their health care provider for weight gain and nutrition information that may not be evidence-based. ${ }^{5,7}$

Interestingly, findings by McDonald et $\mathrm{al}^{5}$ show that more GP and OB patients exceed IOM recommendations than MW patients. Our results show that MWs reported the highest prevalence of counseling in GWG, physical activity, and nutrition when compared to other disciplines, at $93.5 \%$, $96.9 \%$, and $96.9 \%$, respectively. This finding is consistent with that of McDonald et al, ${ }^{5}$ who found that more (64.4\%) women recalled being counseled on GWG by MWs than by GPs (39.0\%) and OBs (35.5\%); this highlights discrepant patient messaging dependent on care provider discipline. Though MWs recommend a slightly greater gestational gain, a higher degree of contact and longer appointments, ${ }^{5}$ and thus more opportunities for counseling during her pregnancy, may help the patient achieve an appropriate GWG target. If physicians and nurses had longer and/or more frequent 
contact with patients, the information overload experienced by pregnant women at each visit may be reduced, thus increasing the chance that they would retain all the GWG information presented. ${ }^{8}$

Recent evidence suggests that, when a provider uses the "5As" counseling approach in weight management, patient motivation to lose weight and intention to change behaviors increase. ${ }^{18}$ Although we are not encouraging weight loss in the pregnant population, employing the 5As may be one tool that can assist providers with GWG management. Lastly, a recent work from Oken et $\mathrm{al}^{20}$ suggests that automatic prompts in the Electronic Medical Records (EMRs) are a very useful tool for counseling and tracking GWG that supports guideline-concordant care. Overall, the 5As, in addition to other, more advanced, electronic messaging prompts through the EMRs, may help improve the frequency and accuracy of GWG tracking and counseling, by not only reminding care providers about GWG, but by allowing them to approach the topic in a manner that limits weight bias.

\section{Health care provider self-perceived knowledge}

A lack of health care provider self-perceived knowledge does not appear to be the problem, as the majority of providers reported feeling that they have adequate knowledge to counsel on GWG, despite few reporting specialized training. However, the translation of knowledge from provider to patient may suggest an area for improvement. In our sample, at least $80 \%$ of providers in each discipline felt confident in their knowledge. Of great interest is that fewer GPs $(86.2 \%)$ felt that they have adequate knowledge to counsel on GWG than the percentage that reported counseling (93.1\%). In contrast, more OBs and MFMs feel that they have the knowledge than actually counsel on these subjects, suggesting that there are barriers to knowledge dissemination that may preclude the patient from receiving optimal care. This suggests that

Table 6 The Canadian Obesity Network (CON) 5As of Obesity Management ${ }^{\mathrm{TM}, 21}$

Ask Ask the patient permission to discuss weight and related issues.

Assess Assess patient risk, behaviors, and readiness to change.

Advise Advise the patient on possible management strategies.

Agree Agree on a treatment plan and collaboratively set goals.

Assist Assist the patient by addressing barriers and securing support. Arrange for follow-up.

Notes: Recently, CON has initiated an international campaign to increase the saliency of the $5 \mathrm{As}$ in clinical weight management as a way to aid the provider and encourage effective and efficient care provision. CON substituted "Arrange" with "Ask" to encourage discussion about weight that minimizes blame and stigmatization. ${ }^{18}$ some inaccurate information may be presented to pregnant patients by GPs. This is potentially compounded if specialists are not providing GWG recommendations and are expecting GPs to give this information. However, we do realize that specialists may not feel that GWG, physical activity, and nutrition counseling is appropriate when caring for high-risk pregnancies with severe complications. Furthermore, when asked who they thought should be providing this information in the event that they did not feel it was their role to do so, $98.6 \%$ of all providers sampled feel that all maternity care providers (GPs, OBs, MWs, etc) should be giving information about physical activity, nutrition, and GWG. However, only $80 \%$ of providers (only two-thirds of OBs) feel that GPs alone should counsel on these topics (see Table 5). It seems that some disciplines believe that others should be discussing GWG, physical activity, and nutrition instead of themselves. Incorporating the $5 \mathrm{As}$ of Obesity Management ${ }^{\mathrm{TM}}$ of the Canadian Obesity Network ${ }^{21}$ may limit interprofessional variation in knowledge transfer (Table 6).

Overall, we have identified a communication gap in maternal care provision. Care providers are unaware who is best suited to articulate information about GWG and thus may not feel that this topic is a primary responsibility of their discipline. This calls for consensus and a need to standardize the provision of this information (who, when, and what) to the patient, ensuring that the information channel is open and also that the patient is receiving the most evidence-based guidance.

\section{Physical activity and nutrition knowledge dissemination}

With respect to knowledge and dissemination of topics pertaining to physical activity and nutrition, a significant discrepancy between disciplines exists ( $P<0.05$ for both). Patients receive different degrees of care dependent on the type of professional from whom they seek care. Roughly $97 \%$ of GPs reported counseling their patients on nutrition in pregnancy, compared to $79.1 \%$ of OBs and $63.6 \%$ of MFMs. It is possible that these values are inflated if health care providers consider solely advising women to take prenatal vitamins as counseling on nutrition, meaning that even fewer providers are advising on healthy eating strategies during pregnancy, which is supported by a previous work by Ferraro et al. ${ }^{7}$ In terms of perceived knowledge, providers feel the least confident in counseling on nutrition, which is alarming, as nutrition during pregnancy is a modifiable lifestyle factor that may help limit cardiometabolic risk in mother and baby. ${ }^{22}$ Thus, many pregnant women may be lacking vital information that could 
contribute to a healthy pregnancy. ${ }^{7}$ While we acknowledge that downstream health outcomes may not be the focal point of clinical discussion, given the time constraints of clinical practice, we believe that employing simple tools (such as $5 \mathrm{As}$ and EMR prompts) will educate women about the longer-term implications of physical activity and healthful nutrition. These tools may serve to reinforce these behaviors in the prenatal and postpartum periods.

\section{Limitations}

Although we attempted to capture a Canadian perspective by utilizing the SOGC mailing list, our study is not without shortcomings. While we were able to delineate whether care providers reported making recommendations, we could not assess the accuracy of reporting nor the quality of the information presented to the patient. Furthermore, given our $15 \%$ response rate, we cannot preclude nonresponse bias, as we may have sampled those most interested in the topic, meaning that the estimates of the proportion of care providers recommending GWG within IOM limits may be higher in our study than in the general target population, thus overestimating accurate obstetric knowledge transfer. However, if this is true and the respondents comprised members most aware of lifestyle counseling and accurately made regular GWG recommendations, then this may bias our results away from the null (ie, providers accurately recommend GWG for patients with obesity), thus strengthening our conclusion if assessing a representative provider population. Nonetheless, we believe that our findings provide an interesting perspective in maternal care provision. Furthermore, with respect to sample size, a recent meta-analysis by Shih and Fan showed that, although the average response rate for email surveys is $33 \%( \pm 22 \%)$, the variability of response rates in their study ranged from 5\%-85\%. ${ }^{23}$ Accordingly, our results fall within one standard deviation of the mean and align with previous research ${ }^{23}$ that has utilized electronic surveys. Lastly, knowing that all providers were aware of the purpose and content of the information being questioned, one cannot rule out the potential influence of the Hawthorne effect (subconscious modification of answers) nor the "open-book exam theory" (consultation of guidelines to verify responses). If this was the case, however, our results provide a conservative estimate of knowledge transfer in obstetrical care.

\section{Conclusion}

Despite previous reports that pregnant patients report very low levels of counseling about GWG, physical activity, and nutrition, ${ }^{5,7}$ the majority of health care providers surveyed reported counseling patients on GWG, physical activity, and nutrition and were able to identify appropriate GWG for patients with obesity. Although it appears that maternity care providers equally value the need to counsel women on GWG, a disconnect exists between what providers report discussing and what, according to the literature currently available, ${ }^{3,4,7}$ patients report receiving. Thus, if providers make recommendations on healthy active living (ie, pregnancy-specific nutrition emphasizing energy balance and physical activity) with a particular focus on attenuating excessive GWG, adverse weight-related outcomes may improve. If time during the prenatal visits restricts the quality of the advice given, incorporating allied health professionals who specialize in these topics into the care team may be an appropriate model in an attempt to offset maternal and fetal pathologies that are largely preventable.

\section{Acknowledgments}

The authors wish to thank the Ottawa Dragon Boat Foundation and the SOGC staff and members for their support with this project.

\section{Disclosure}

The authors report no conflicts of interest in this work.

\section{References}

1. Adamo KB, Ferraro ZM, Brett KE. Can we modify the intrauterine environment to halt the intergenerational cycle of obesity? Int J Environ Res Public Health. 2012;9(4):1263-1307.

2. Phelan S, Phipps MG, Abrams B, Darroch F, Schaffner A, Wing RR. Practitioner advice and gestational weight gain. $J$ Womens Health (Larchmt). 2011;20(4):585-591.

3. Lutsiv O, Bracken K, Pullenayegum E, Sword W, Taylor VH, McDonald SD. Little congruence between health care provider and patient perceptions of counselling on gestational weight gain. $J$ Obstet Gynaecol Can. 2012;34(6):518-524.

4. McDonald SD, Pullenayegum E, Taylor VH, et al. Despite 2009 guidelines, few women report being counseled correctly about weight gain during pregnancy. Am J Obstet Gynecol. 2011;205(4):333-336.

5. McDonald SD, Pullenayegum E, Bracken K, et al. Comparison of midwifery, family medicine, and obstetric patients' understanding of weight gain during pregnancy: a minority of women report correct counselling. J Obstet Gynaecol Can. 2012;34(2):129-135.

6. Herring SJ, Platek DN, Elliott P, Riley LE, Stuebe AM, Oken E. Addressing obesity in pregnancy: what do obstetric providers recommend? J Womens Health (Larchmt). 2010;19(1):65-70.

7. Ferraro ZM, Rutherford J, Keely E, Dubois L, Adamo KB. An assessment of patient information channels and knowledge of physical activity and nutrition during pregnancy. Obstet Med. 2011;4:59-65.

8. Kessels RP. Patients' memory for medical information. $J$ R Soc Med. 2003;96(5):219-222.

9. Sui Z, Grivell RM, Dodd JM. Antenatal exercise to improve outcomes in overweight or obese women: a systematic review. Acta Obstet Gynecol Scand. 2012;91(5):538-545.

10. Tanentsapf I, Heitmann BL, Adegboye AR. Systematic review of clinical trials on dietary interventions to prevent excessive weight gain during pregnancy among normal weight, overweight and obese women. BMC Pregnancy Childbirth. 2011;11:81. 
11. Artal R, Lockwood CJ, Brown HL. Weight gain recommendations in pregnancy and the obesity epidemic. Obstet Gynecol. 2010;115(1): 152-155.

12. Sharma AM, Kushner RF. A proposed clinical staging system for obesity. Int J Obes (Lond). 2009;33(3):289-295.

13. Padwal RS, Pajewski NM, Allison DB, Sharma AM. Using the Edmonton obesity staging system to predict mortality in a populationrepresentative cohort of people with overweight and obesity. CMAJ. 2011;183(14):E1059-E1066.

14. Alanis MC, Goodnight WH, Hill EG, Robinson CJ, Villers MS, Johnson DD. Maternal super-obesity (body mass index $>$ or $=50$ ) and adverse pregnancy outcomes. Acta Obstet Gynecol Scand. 2010;89(7): 924-930.

15. Butte NF. Energy requirements during pregnancy and consequences of deviations from requirement on fetal outcome. Nestle Nutr Workshop Ser Pediatr Program. 2005;55:49-67.

16. Butte NF, King JC. Energy requirements during pregnancy and lactation. Public Health Nutr. 2005;8(7A):1010-1027.

17. Catalano PM, Presley L, Minium J, Hauguel-de Mouzon S. Fetuses of obese mothers develop insulin resistance in utero. Diabetes Care. 2009;32(6):1076-1080.
18. Jay M, Gillespie C, Schlair S, Sherman S, Kalet A. Physicians' use of the 5As in counseling obese patients: is the quality of counseling associated with patients' motivation and intention to lose weight? $B M C$ Health Serv Res. 2010;10:159.

19. Davies GA, Maxwell C, McLeod L. Obseity in pregnancy. J Obset Gynaecol Can. 2010;32(2):165-173.

20. Oken E, Switkowski K, Price S, et al. A qualitative study of gestational weight gain counseling and tracking. Matern Child Health J. Epub October 14, 2012.

21. 5As of Obesity Management ${ }^{\mathrm{TM}}$ [webpage on the Internet]. Edmonton, AB: Canadian Obesity Network. Available from: http://www.obesitynetwork.ca/5As). Accessed June 17, 2012.

22. Adamo KB, Ferraro ZM, Brett KE. Pregnancy is a critical period for prevention of obesity and cardiometabolic risk. Can J Diabetes. 2012;36:134-141.

23. Shih TH, Fan $X$. Comparing response rates in email and paper surveys: a meta-analysis. Educational Research Review. 2009;4:26-40.
International Journal of Women's Health

\section{Publish your work in this journal}

The International Journal of Women's Health is an international, peerreviewed open-access journal publishing original research, reports, editorials, reviews and commentaries on all aspects of women's healthcare including gynecology, obstetrics, and breast cancer. The manuscript management system is completely online and includes

\section{Dovepress}

a very quick and fair peer-review system, which is all easy to use Visit http://www.dovepress.com/testimonials.php to read real quotes from published authors. 